\title{
The self-focusing condition of a charged particle beam in a resistive plasma
}

\author{
Xiao-Chuan Ning ${ }^{1}$, Tian-Yi Liang ${ }^{1}$, D. Wu ${ }^{1,2, \dagger}$ and Zheng-Mao Sheng ${ }^{(1,} \dagger$ \\ ${ }^{1}$ Institute for Fusion Theory and Simulation, Department of Physics, Zhejiang University, Hangzhou, \\ 310027, PR China \\ ${ }^{2}$ Collaborative Innovation Center of IFSA, Shanghai Jiao Tong University, Shanghai, 200240, PR China
}

(Received 10 March 2021; revised 26 September 2021; accepted 28 September 2021)

The self-focusing condition of a charged particle beam in a resistive plasma has been studied. When plasma heating is weak, the beam focusing is intensified by increasing the beam density or velocity. However, when plasma heating is strong, the beam focusing is only determined by the beam velocity. Especially, in weak heating conditions, the beam trends to be focused into the centre as a whole, and in strong heating conditions, a double-peak structure with a hollow centre is predicted to appear. Furthermore, it is found that the beam radius has a significant effect on focusing distance: a larger the beam radius will result in a longer focusing distance. Simulation results also show that when the beam radius is large enough, filamentation of the beam appears. Our results will serve as a reference for relevant beam-plasma experiments and theoretical analyses, such as heavy ion fusion and ion-beam-driven high energy density physics.

Key words: particle/fluid flow, nonlinear instability, plasmas

\section{Introduction}

Transport of a charged particle beam in a background plasma is the foundation for a variety of applications, such as inertial confinement fusion (Roth et al. 2001; Logan, Perkins \& Barnard 2008; Frolova, Khishchenko \& Charakhch'yan 2019), high-energy particle accelerators and colliders (Govil et al. 1999), radiography (Sheng et al. 2014), astrophysics (Bennett 1934; Alfvén 1939; Spitkovsky 2007) and material etching (Lami et al. 2020). Within the transport, the beam can strongly interact with background plasmas, potentially causing many physical phenomena, commonly including exciting periodic plasma wake-fields (Adli et al. 2018; Caldwell et al. 2018; Martinez de la Ossa, Mehrling \& Osterhoff 2018; Turner et al. 2019) and beam-plasma instabilities (Lee \& Lampe 1973; Davidson et al. 2004; Tokluoglu et al. 2018). In addition, beam focusing may also be induced under some circumstances, which has aroused our great interest.

On the one hand, beam focusing may occur in all kinds of phenomena associated with beam-plasma physics as mentioned above. Studying it will deepen our understanding

†Email addresses for correspondence: dwu.phys@ zju.edu.cn, zmsheng@zju.edu.cn 
of many beam-plasma physical processes. On the other hand, beam focusing also has a wide application prospect. This is because in many fields, such as heavy ion fusion and ion-beam-driven high energy density physics (Callahan 1996; Logan et al. 2007), a focused beam is of high energy density, which leads to a higher efficiency of target heating. Therefore, beam focusing deserves our in-depth research.

A large amount of important work has previously been conducted on this topic (Bennett 1934, 1955; Robertson 1982; Kaganovich et al. 2001; Roy et al. 2005; Dorf et al. 2009; Seidl et al. 2009; Dorf et al. 2011, 2012; Robinson, Key \& Tabak 2012; Hu \& Wang 2015; Chen et al. 2020). Approximately a century ago, Bennett published two papers on the magnetically self-focusing relation of mixed streams consisting of electrons and ions (Bennett 1934, 1955), which, to the best of our knowledge, are the pioneering work in this field. Even after such a long time, focusing was remained a hot topic, especially in the last twenty years. For example, Kaganovich et al. (2001) found that for a cold long ion beam, when propagating in a background plasma where plasma electrons are cold and electron thermal effects are neglected, its charge can be well neutralized by plasma electrons and the beam can then be focused by the dominant self-generated magnetic field. Roy et al. (2005) experimentally achieved longitudinal compression of an intense neutralized ion beam by providing a head-to-tail velocity tilt. Dorf et al. (2012) focused an ion beam by first passing it through a background plasma, extracting enough neutralizing electrons and then making it enter a magnetic lens. Though many studies on beam focusing have been performed, the focusing condition of a charged particle beam propagating in a resistive plasma is still not clear both in theory or experiment. Recently, we carried out detailed theoretical analysis and simulation research on this topic and achieved some meaningful results. Both theoretical analysis and simulations show that the focusing strongly depends on the beam density, beam velocity and beam radius. We believe that this study will provide useful ideas for relevant experiments and theoretical exploration on beam-plasma physics.

The paper is organized as follows. Section 2 introduces the theoretical model and gives the specific self-focusing condition. Furthermore, the influences of beam parameters on focusing are also discussed. In $\S 3$, simulation results are presented and compared with theoretical analysis. In $\S 4$, our conclusions are finally submitted.

\section{Theory}

Before the theory is introduced, two aspects should be emphasized in advance. The first is that when a charged particle beam is injected into a resistive plasma, we assume the beam is quickly neutralized by plasma electrons. This assumption holds as the beam duration time is much longer than the electron plasma period $2 \pi / \omega_{\mathrm{pe}}\left(\omega_{\mathrm{pe}}\right.$ is the electron plasma frequency) and the beam radius is much larger than the plasma electron skin depth $c / \omega_{\mathrm{pe}}$ ( $c$ is the light speed) (Kaganovich et al. 2001, 2008; Berdanier, Roy \& Kaganovich 2015). The second aspect is that the focusing we are describing here is the transverse compression of the entire beam. As shown in figure 1, after entering the plasma, the beam is in a state of unbalanced force in the transverse direction, which causes its radius to gradually become smaller and at the same time, its density increases, which lead to the focusing. To intuitively explain this phenomenon and determine the focusing condition, we need to figure out the major forces acting on the beam, so we might as well start with a force analysis of the beam. When a charged particle beam propagates in a uniform background plasma, its motion equation can be written as

$$
n_{b} \frac{\mathrm{d} p_{b}}{\mathrm{~d} t}=-\nabla p_{\text {th }}+\boldsymbol{J}_{b} \times \boldsymbol{B}+n_{b} q \boldsymbol{E} .
$$




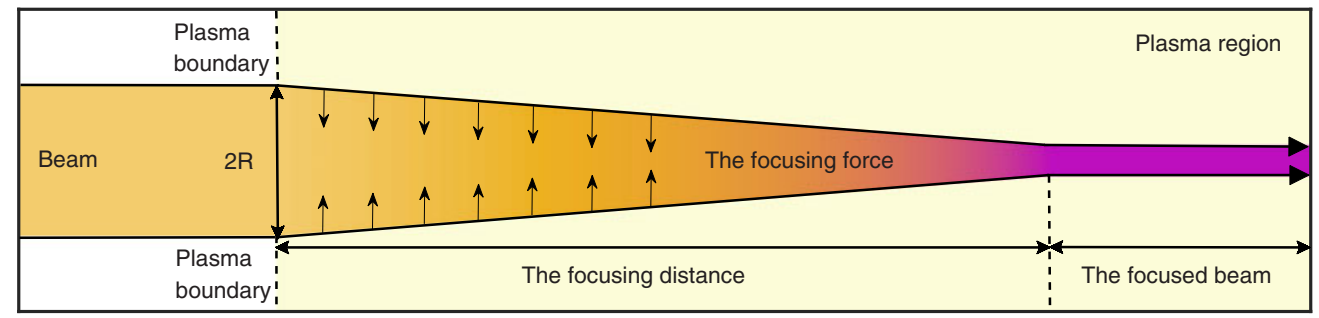

FIGURE 1. A schematic diagram of beam focusing. A beam, whose spot size is $2 R$, is injected from the left-hand side of the box into a background plasma. During the transport, the beam radius gradually becomes smaller owing to the focusing force and after the focusing distance, the beam finally gets focused.

On the left-hand side of the equation, $n_{b}$ is the density of the beam and $\boldsymbol{p}_{b}$ is the momentum of the beam particle; on the right-hand side, $p_{\text {th }}$ is the beam thermal pressure and can be estimated from the equation of state $p_{\text {th }}=n_{b} k T_{b}$, where $k$ is Boltzmann's constant and $T_{b}$ is the beam temperature, $\boldsymbol{J}_{b}$ is the beam current density, $q$ is the effective charge of a beam particle, $\boldsymbol{B}$ is the magnetic field and $\boldsymbol{E}$ is the electric field. According to the basic Ohm's law, the electric field $\mathrm{E}$ can be expressed as

$$
\boldsymbol{E}=\eta \boldsymbol{J}_{e}-\boldsymbol{v}_{e} \times \boldsymbol{B}-\frac{1}{e n_{e}} \nabla p_{e},
$$

where $\eta$ is the resistivity of the background plasma, considered to obey Spitzer formulation, $\eta \propto T^{-3 / 2}, \boldsymbol{J}_{e}$ is the return current density of plasma electrons, $\boldsymbol{v}_{e}$ is the flow velocity of plasma electrons, $e$ is the elementary charge. Additionally, $n_{e}$ and $p_{e}$ are the density and the thermal pressure of plasma electrons, respectively. In Cartesian coordinates $(x, y, z)$, considering the beam moving in the $z$ direction, when the transverse density distribution is assumed to be Gaussian profile, we can express its current density as $\boldsymbol{J}_{b}=n_{b} q v_{b} \boldsymbol{e}_{z}=n_{b 0} q v_{b} \exp \left(-\left(x^{2}+y^{2}\right) / R^{2}\right) \boldsymbol{e}_{z}$, where $n_{b 0}$ is the central density of the beam, $v_{b}$ is the beam velocity and $R$ is the beam radius. Here, to illustrate our study method, we only take the $y$ direction as an example, and therefore, it is not necessary to consider the density distribution in the $x$ direction. For simplicity, we assume plasma ions to be at rest. Then, with the application of the beam charge and current neutralization, we have $n_{e} \approx n_{p}+(q / e) n_{b}$ and $\boldsymbol{J}_{e} \approx-\boldsymbol{J}_{b}$, where $n_{p}$ is the initial density of plasma electrons. We assume the charge density of the beam is much smaller than that of the plasma. Therefore, we can obtain $\boldsymbol{v}_{e} \ll \boldsymbol{v}_{b}$. The thermal pressure $p_{e}$ can also be estimated from the equation of state $p_{e}=n_{e} k T_{0}$, where $T_{0}$ is the plasma temperature. Then, the electric field can be written as

$$
\boldsymbol{E} \approx-\eta J_{b}-\boldsymbol{v}_{e} \times \boldsymbol{B}-\frac{q k T_{0}}{e^{2} n_{e}} \nabla n_{b} .
$$

For the beam, the forces of the electric field in the transverse direction, i.e. the second and the third terms on the right-hand side of (2.3), can be considered as small quantities compared with the Lorentz force $J_{b} \times B$ and the beam thermal pressure $\nabla p_{\text {th }}$. This is because of $\left|n_{b} q \boldsymbol{v}_{e} \times \boldsymbol{B}\right| /\left|\boldsymbol{J}_{b} \times \boldsymbol{B}\right| \approx v_{e} / v_{b} \ll 1$ and $\left|n_{b} q^{2} k T_{0} \nabla n_{b} /\left(e^{2} n_{e}\right)\right| /\left|\nabla p_{\mathrm{th}}\right| \approx$ $\left|n_{b} q^{2} T_{0}\right| /\left|n_{e} e^{2} T_{b}\right| \ll 1$, where we have assumed the beam temperature is not much lower than the plasma temperature, $T_{b} \lesssim T_{0}$. Therefore, we might approximately express the electric field as $E \approx-\eta J_{b}$. Hence, the motion of the beam in the $y$ direction is constrained by the thermal pressure $p_{\text {th }}$ and the Lorentz force $J_{b} B_{x}$. The motion equation in the $y$ 


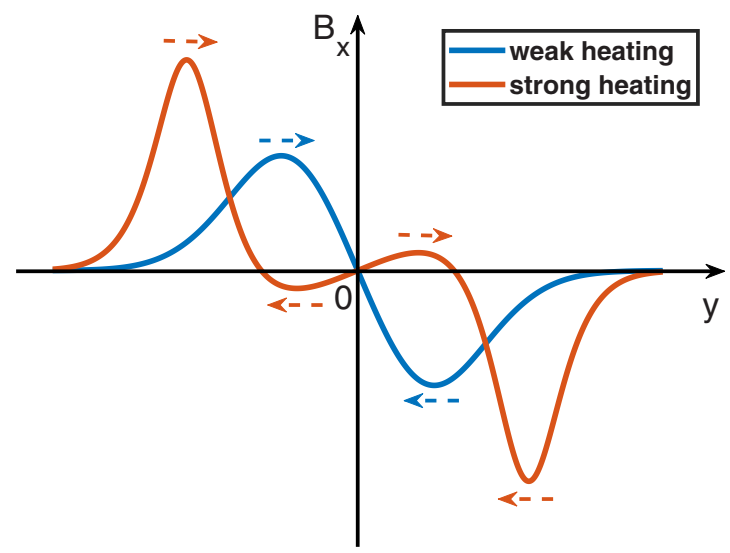

FIGURE 2. A schematic diagram of the magnetic field distributions for weak heating (blue solid line) and strong heating (red solid line). The blue and red dashed arrows indicate the force direction of the Lorentz force for weak heating and strong heating, respectively.

direction further becomes

$$
n_{b} \frac{\mathrm{d} p_{b y}}{\mathrm{~d} t}=-\frac{\mathrm{d} p_{\mathrm{th}}}{\mathrm{d} y}+J_{b} B_{x},
$$

where $p_{b y}$ is the component of the beam momentum in the $y$ direction. The magnetic field $B_{x}$ can be derived from Faraday's law:

$$
\frac{\partial \boldsymbol{B}}{\partial t}=-\nabla \times \boldsymbol{E}=\nabla \times\left(\eta \boldsymbol{J}_{b}\right) .
$$

This model for solving the electric field and the magnetic field has been widely used by many researchers (Davies et al. 1997; Bell, Davies \& Guerin 1998; Davies 2003; Davies, Green \& Norreys 2006; Robinson et al. 2012; Norreys et al. 2014; Kim et al. 2016; Curcio \& Volpe 2019). For example, to simplify calculations and obtain a specific analytical form of the fields, Davies considered a rigid beam model (Davies 2003). Using this model, the plasma temperature is increased by Ohmic heating, $\partial T / \partial t=2 \eta J_{b}^{2} /\left(3 k n_{e}\right)$ and the resistivity also changes accordingly, $\eta=\eta_{0}\left(T / T_{0}\right)^{-3 / 2}$, where we have applied the Spitzer resistivity formulation. Then combining these two relations with (2.5) and doing some integral operations, we can get the magnetic field. Making use of Davies' conclusion (Davies 2003), we can express the magnetic field as

$$
B_{x}=\frac{\mathrm{d} J_{b}}{\mathrm{~d} y} \frac{3 n_{e} k T_{0}}{2 J_{b}^{2}}\left[1-\frac{1}{5}(1+\Omega)^{2 / 5}-\frac{4}{5}(1+\Omega)^{-3 / 5}\right],
$$

where

$$
\Omega=\frac{5}{3} \frac{\eta_{0} J_{b}^{2} \tau}{n_{e} k T_{0}}
$$

and $\tau=t-z / v_{b}$ is the time variable. Here, $\Omega$ means the ratio of Ohmic heating energy to plasma electrons thermal energy and $\Omega_{\max }$ is located at $y=0$. For $\Omega_{\max } \ll 1$, the plasma temperature is slightly increased, which corresponds to weak heating, and for $\Omega_{\max } \gg 1$, the plasma temperature is significantly increased, which corresponds to strong heating. A schematic diagram of the magnetic field distributions in the cases of weak heating 
and strong heating is shown in figure 2. For weak heating, the magnetic field has a pure focusing effect; and for strong heating, the case is different: the magnetic field near the centre changes its sign and will push the beam outward, while there exists a magnetic field away from the centre that will push the beam inward. This may lead to a result where the beam becomes hollowed near the centre and focused away from the centre. To determine the position where the magnetic field changes its sign, we can solve the following equation:

$$
1-\frac{1}{5}(1+\Omega)^{2 / 5}-\frac{4}{5}(1+\Omega)^{-3 / 5}=0
$$

of which the numerical solution is $\Omega \approx 44$, and for strong heating, the beam focusing can only occur in the range of $0<\Omega<44$. The beam focusing depends on the relation between the beam thermal pressure $-\left(\mathrm{d} p_{\text {th }} / \mathrm{d} y\right)$ and the Lorentz force $J_{b} B_{x}$. They have the forms as follows:

$$
-\frac{\mathrm{d} p_{\mathrm{th}}}{\mathrm{d} y}=-k T_{b} \frac{\mathrm{d} n_{b}}{\mathrm{~d} y}
$$

and

$$
J_{b} B_{x}=\frac{\mathrm{d} J_{b}}{\mathrm{~d} y} \frac{3 n_{e} k T_{0}}{2 J_{b}}\left[1-\frac{1}{5}(1+\Omega)^{2 / 5}-\frac{4}{5}(1+\Omega)^{-3 / 5}\right] .
$$

The specific focusing condition can be obtained by solving

$$
\left|J_{b} B_{x}\right|>\left|-\frac{\mathrm{d} p_{\mathrm{th}}}{\mathrm{d} y}\right| \text {. }
$$

For weak heating, the Lorentz force can be simplified as

$$
J_{b} B_{x} \approx \eta_{0} J_{b} \frac{\mathrm{d} J_{b}}{\mathrm{~d} y} \tau
$$

and the focusing condition is

$$
\frac{n_{b} q^{2} v_{b}^{2}}{k T_{b}}>\frac{1}{\eta_{0} \tau}
$$

For strong heating, in the range of $\Omega \gg 1$ (at least $\Omega>44$ ), where $|y| \ll \sqrt{\ln \Omega_{\max } / 2} R$, the Lorentz force can be approximately written as

$$
J_{b} B_{x} \approx-\frac{1}{2} \eta_{0} J_{b} \frac{\mathrm{d} J_{b}}{\mathrm{~d} y} \tau \Omega^{-3 / 5}
$$

which, compared with (2.12), has an opposite sign and will cause beam hollowing (Davies 2003; Davies et al. 2006), as discussed above. In the range of $0<\Omega<44$, there is a strong focusing magnetic field, which potentially can focus the beam. To get an accurate focusing condition in this range, we have to solve inequality (2.11). For the sake of simplicity and ease of use, we can estimate the order of magnitude of the Lorentz force by letting $\Omega \approx 1$, which corresponds to $|y| \approx \sqrt{\ln \Omega_{\max } / 2} R$. Based on this position and making use of (2.9) and (2.10) and inequality (2.11), we can get an approximate focusing condition for strong heating,

$$
\frac{q v_{b}}{k T_{b}}>\sqrt{\frac{1}{\eta_{0} \tau n_{e} k T_{0}}},
$$

where for simplicity, we have discarded the constant coefficient. The above results in inequalities (2.13) and (2.15) show that in the weak heating regime, increasing either beam 
density or velocity is good for focusing and in the strong heating regime, only increasing the beam velocity facilitates focusing. It should be mentioned that in the theory, we have neglected the effect of the magnetic diffusion, where the time scale is estimated by

$$
\tau_{m d}=\frac{\mu_{0} R^{2}}{\eta},
$$

where $\mu_{0}$ is the vacuum permeability. The resistivity is large for bad conductors, but usually is small for ionized plasmas. For widely used electron and ion beams generated by intense lasers, their duration time is much shorter than the magnetic diffusion time. In these cases, it is reasonable to ignore the magnetic diffusion. Next, we will discuss the effect of the beam radius $R$ on focusing. Adding (2.9) and (2.10), we can express the resultant force $f_{y}$ as

$$
f_{y}=-\frac{\mathrm{d} p_{\mathrm{th}}}{\mathrm{d} y}+J_{b} B_{x}=\frac{y}{R^{2}} F_{y}
$$

where

$$
F_{y}=-3 n_{e} k T_{0}\left[1-\frac{1}{5}(1+\Omega)^{2 / 5}-\frac{4}{5}(1+\Omega)^{-3 / 5}\right]+2 n_{b} k T_{b} .
$$

Once the relative position of the beam is determined, the resultant force $f_{y}$ is proportional to $1 / R, f_{y} \propto 1 / R$. According to this relation, we are able to draw a conclusion that if a charged particle beam can be focused, a larger radius $R$ will lead to a smaller resultant force $f_{y}$, a longer focusing time and a longer focusing distance.

Similar focusing conclusions have also been obtained by other researchers (Bennett 1934, 1955; Kaganovich et al. 2001; Dorf et al. 2009, 2012). Considering a mixed stream of counterstreaming ions and electrons, Bennett derived the total focusing force by summing over the force of a single particle on another single particle and found that to make the stream focused, the total current should exceed a critical value to overcome transverse spread arising from transverse velocity components (Bennett 1934, 1955). Based on cold plasma electron fluid equations with Maxwell's equations, assuming an exact charge neutralization of an ion beam by background plasma electrons, Kaganovich et al. (2001) obtained the focusing force which depends on the degree of the current neutralization and it was found that if the radial ion thermal velocity is ignored, the beam can always be focused (Kaganovich et al. 2001).

Though to some extent, our conclusions appear to be alike, the physical image we describe and the model we use to calculate the electromagnetic fields in this paper are different. In our model, we treat the plasma as a conductor with a resistivity and assume the beam can be well neutralized by plasma electrons. Because Ohmic heating is considered, the plasma temperature will be increased and plasma resistivity will be decreased. According to the Ohm's law and Faraday's law, the generated magnetic field is not static and will evolve in response to changes in plasma resistivity. Likewise, the focusing force and the focusing condition are also not constant, but vary with the degree of heating.

\section{Simulation}

To confirm the above theoretical predictions, we have used LAPINS code (Chen et al. 2020; Ren et al. 2020; Wu et al. 2018, 2020) to make multiple sets of 2D3V simulations of a proton beam propagating in a uniform large-scale hydrogen plasma. As we know for typical particle-in-cell (PIC) simulations, the plasma frequency needs to be resolved, and moreover the grid size must be comparable to the Debye length to minimize artificial 


$\begin{array}{cccccc}n_{p}\left(n_{0}\right) & v_{p 1}(c) & v_{p 2}(c) & v_{p 3}(c) & v_{p 4}(c) & v_{p 5}(c) \\ 0.05 & 0.10 & 0.14 & 0.20 & 0.31 & 0.43 \\ 0.1 & 0.05 & 0.10 & 0.14 & 0.20 & 0.31 \\ 0.5 & 0.05 & 0.10 & 0.13 & 0.20 & 0.31 \\ 1.0 & 0.05 & 0.10 & 0.13 & 0.20 & 0.31 \\ 5.0 & 0.05 & 0.10 & 0.14 & 0.20 & 0.31 \\ 10.0 & 0.03 & 0.05 & 0.14 & 0.20 & 0.31\end{array}$

TABLE 1. In the 30 sets of simulations run by LAPINS code, the values of the proton beam density (in units of $n_{0}$ ) and velocity (in units of the light speed $c \approx 3.0 \times 10^{8} \mathrm{~m} \mathrm{~s}^{-1}$ ). The first column is the proton beam density parameters, and the second to the sixth columns are the proton beam velocity parameters at different densities.

grid heating and suppress numerical instabilities. Therefore, high noise levels and high computational requirements arising from the operation on the shortest time and length scales greatly limit the applications of PIC methods to large-scale simulations.

To efficiently investigate the transport of intense charged particles, the significant update to the LAPINS code has recently been made. For our interests, the current density of proton beams is so high that electromagnetic effects need to be considered. However, when compared with the density of the plasma target, the current density of the proton beam is still several orders of magnitude lower. For this particular case, instead of solving the full Maxwell's equations, we couple Ampere's law, Faraday's law and Ohm's law to update the electric and magnetic fields. To be specific, plasma ions and the injected beam particles are treated by the PIC method, while plasma electrons are treated as a fluid, which is solved by Ampere's law, $\boldsymbol{J}_{e}=(1 / 2 \pi) \boldsymbol{\nabla} \times \boldsymbol{B}-(1 / 2 \pi)(\partial \boldsymbol{E} / \partial t)-\boldsymbol{J}_{b}-\boldsymbol{J}_{i}$, where $\boldsymbol{B}$ is the magnetic field, $E$ is the electric field, $\boldsymbol{J}_{b}$ is the beam current and $\boldsymbol{J}_{i}$ is the plasma ion current. The electric field is obtained by Ohm's law, $\boldsymbol{E}=\eta \boldsymbol{J}_{e}-\boldsymbol{v}_{e} \times \boldsymbol{B}-\nabla p_{e} / e n_{e}$, where $\eta$ is the resistivity, $\boldsymbol{v}_{e}$ is the flow velocity of plasma electrons, $p_{e}$ is the plasma electron pressure, $n_{e}$ is the plasma electron density and $e$ is the elementary charge. The magnetic field is obtained by Faraday's law, $\partial \boldsymbol{B} / \partial t=-\boldsymbol{\nabla} \times \boldsymbol{E}$. As only a part of Maxwell's equations needs to be solved, this method is quick, which is useful for large-scale simulations. Furthermore, collisional effects are included based on a Monte Carlo binary collision model, which can deal well with calculating the beam stopping, plasma heating and thermal conduction.

In the simulations, the size of a cell is $0.1 \mathrm{~mm} \times 0.1 \mathrm{~mm}$ and the time step is $0.17 \mathrm{ps}$, the plasma temperature is $4 \mathrm{eV}$ and density is $10^{5} n_{0}$, where $n_{0}=1.1 \times 10^{19} \mathrm{~m}^{-3}$. The beam temperature is $4 \mathrm{eV}$. The beam moves in the $z$ direction, where the transverse density distribution is a Gaussian profile in the $y$ direction and the radius is $1 \mathrm{~mm}$. The time variable $\tau$ is set to $0.6 \mathrm{~ns}$. In the case with the above parameters kept fixed, we change the beam density $n_{p}$ and velocity $v_{p}$, and then investigate their propagations in the plasma. The specific density $n_{p}$ and velocity $v_{p}$ of the proton beam vary, as shown in table 1 . Under these conditions, the plasma is weakly heated. The length along the $y$ direction is $5 \mathrm{~mm}$. The length along the $z$ direction is several hundred millimetres. It is not fixed because the focusing distance changes as the beam parameters change.

Figure 3 shows four sets of typical simulation results, from which, we can roughly judge the influences of the beam density, velocity and radius on focusing. In figure 3(a), we see that if the beam density is $1 n_{0}$ and velocity is $0.05 c$, it cannot become focused. However, when we increase its density to $10 n_{0}$, it is found in figure $3(b)$ that the beam can become focused. Similarly, when we increase its velocity to $0.31 c$, as shown in figure $3(c)$, the 

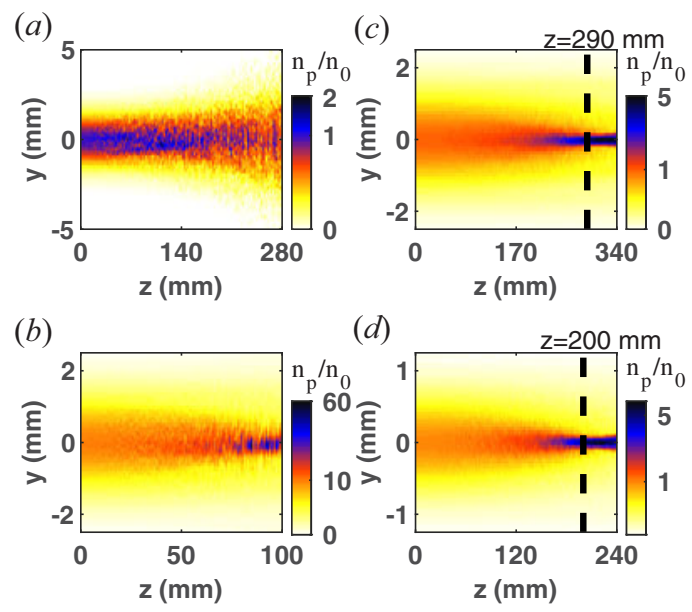

FIGURE 3. Beam densities distributions in four sets of simulations under different beam parameters. Initial densities of the beams are $1 n_{0}$ for $(a, c, d)$ and $10 n_{0}$ for $(b)$. Initial velocities of the beams are $0.05 c$ for $(a, b)$ and $0.31 c$ for $(c, d)$. Initial radii of the beams are $1 \mathrm{~mm}$ for $(a-c)$ and $0.5 \mathrm{~mm}$ for $(d)$. The dashed lines indicate $z=290 \mathrm{~mm}$ for $(c)$ and $z=200 \mathrm{~mm}$ for $(d)$, which are the positions at which the beams eventually become focused.

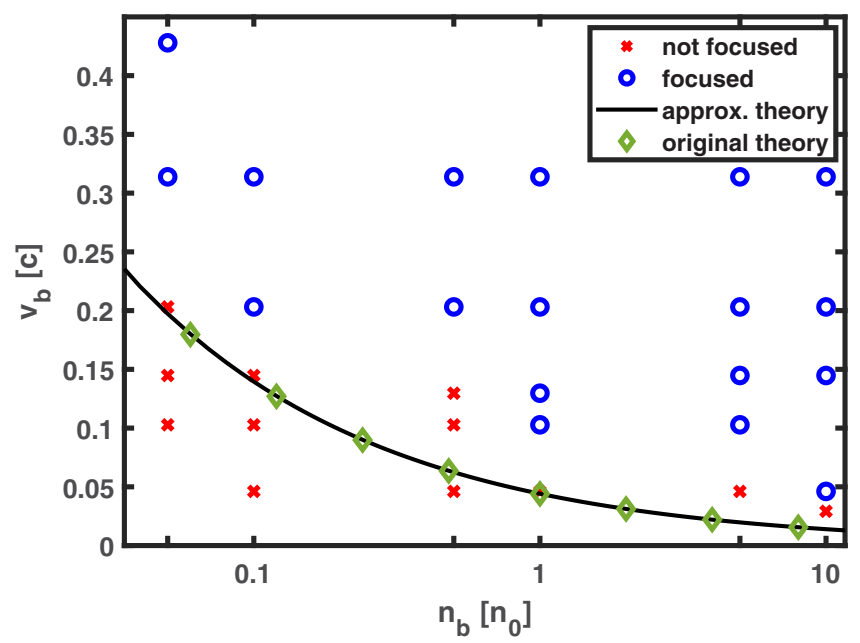

FIgURE 4. Simulation and theoretical results. The red crosses represent that the beam is not focused and the blue circles represent that the beam is focused. The black solid line represents the numerical results solved by $\left(n_{b} q^{2} v_{b}^{2}\right) / k T_{b}=1 / \eta_{0} \tau$ (corresponding to focusing condition (2.13)) at $y=R$. The green diamonds are obtained by the original theory, $\left|J_{p} B_{x}\right|_{\text {theory }}=\left|-\left(\mathrm{d} p_{\text {th }} / \mathrm{d} y\right)\right|$ at $y=R$.

beam also gets focused. Comparing figures $3(c)$ and $3(d)$, we notice that in the case where the other parameters are kept fixed, the focusing distance is different at different radii. In figure 3(c) where the beam radius is $1 \mathrm{~mm}$, the focusing distance is $290 \mathrm{~mm}$, while in figure $3(d)$ where the beam radius is reduced to $0.5 \mathrm{~mm}$, the focusing distance is shortened to $200 \mathrm{~mm}$. 


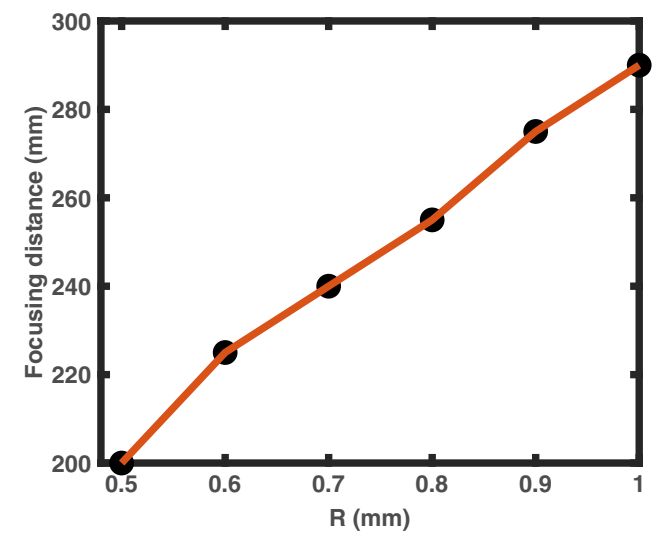

FIGURE 5. Variation of the focusing distance as the beam radius $R$ varies.
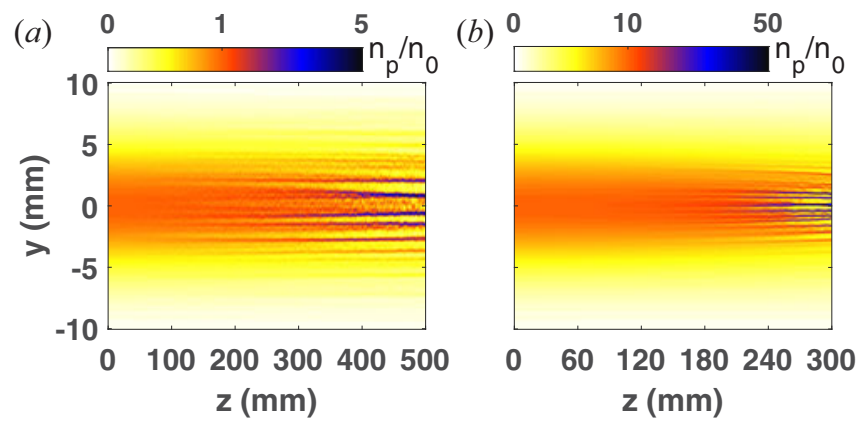

FIGURE 6. Densities distributions of the proton beams in the case of $R=4 \mathrm{~mm}$. Initial densities and velocities of the proton beams are $(a) n_{p}=1 n_{0}, v_{p}=0.31 c$ and $(b) n_{p}=10 n_{0}, v_{p}=0.87 c$, respectively.

The simulation results under table 1 parameters and the theoretical results are displayed in figure 4. If $n_{p}$ and $v_{p}$ are above the green diamonds, it implies $\left|J_{p} B_{x}\right|>\left|-\left(\mathrm{d} p_{\text {th }} / \mathrm{d} y\right)\right|$ and the proton beam can be focused. If $n_{p}$ and $v_{p}$ are below the green diamonds, it implies $\left|J_{p} B_{x}\right|<\left|-\left(\mathrm{d} p_{\mathrm{th}} / \mathrm{d} y\right)\right|$ and the proton beam cannot be focused. Qualitatively, as predicted by the theory in $\S 2$, in the weak heating regime, increasing the proton beam density or velocity is beneficial to focusing. The simulation results also conform to this law. For example, we can clearly see in figure 4 that when the proton beam density $n_{p}$ is set to $0.05 n_{0}$, if the beam velocity $v_{p}$ is less than or equal to $0.20 c$, the beam cannot be focused. With the increase of $v_{p}$, the proton beam can be focused only when $v_{p}$ is greater than or equal to $0.31 c$. However, when the proton beam velocity $v_{p}$ is set to $0.10 c$, if the beam density $n_{p}$ is less than or even equal to $0.5 n_{0}$, the beam cannot be focused. Only when $n_{p}$ is greater than or equal to $1.0 n_{0}$ can the beam be focused.

There is a little difference between our theory and simulations. This may be caused by two aspects. First, for simplicity, in the theory, we considered the beam temperature to be a constant, while actually, in the process of the beam being focused, collisions between beam particles will be enhanced, which can increase the beam temperature and as a result, the beam thermal pressure will also be increased. Second, in the theory, the proton beam velocity $v_{p}$ is considered to be a constant. However in the simulations, owing to collisions and collective electromagnetic effects, the beam is gradually slowed down 
while propagating (Li \& Petrasso 1993; Brown, Dean \& Singleton 2005; Zylstra et al. 2015; Clauser \& Arista 2018; Ren et al. 2020). Therefore, the real velocity of the proton beam in the simulations will be less than the initial theoretical velocity, $v_{\text {sim }}<v_{\text {theory }}$. Then from (2.12), it is easy to prove that the Lorentz force in the simulations will be smaller than the theoretical one, $\left|J_{p} B_{x}\right|_{\operatorname{sim}}<\left|J_{p} B_{x}\right|_{\text {theory }}$. In this way, the theoretical line $\left|J_{p} B_{x}\right|_{\text {theory }}=\left|-\left(\mathrm{d} p_{\text {th }} / \mathrm{d} y\right)\right|$ will be located in the range of $\left|J_{p} B_{x}\right|_{\operatorname{sim}}<\left|-\left(\mathrm{d} p_{\text {th }} / \mathrm{d} y\right)\right|$.

From figures $3(c)$ and $3(d)$, we can roughly judge the relation between the focusing distance and the beam radius. To further test the influence of the beam radius $R$ on focusing, we have done more simulations using LAPINS code. The parameters of the background plasma remain unchanged, and the parameters of the incident proton beam are a density of $1 n_{0}$, velocity of $0.31 \mathrm{c}$ and temperature of $4 \mathrm{eV}$. The value of the beam radius $R$ varies from $0.5 \mathrm{~mm}$ to $1.0 \mathrm{~mm}$. The simulation results are shown in figure 5 . Obviously, as $R$ increases, the focusing distance of the proton beam gradually becomes longer. This law is also consistent with the previous theoretical predictions.

The double-peak focusing structure predicted by the theory in the strong heating regime has not been observed yet. This may be caused by thermal conduction arising from collisions. Moreover, we found that if we further set the beam radius $R$ to a much larger number, such as $4 \mathrm{~mm}$, as shown in figure 6 , the proton beam filamentation phenomenon would occur. There have been many important discoveries and conclusions on electron beam filamentation (Benford 1973; Lee \& Lampe 1973; Bret, Firpo \& Deutsch 2005; Allen et al. 2012; Wang, Hu \& Wang 2020), but ion beam filamentation has not been thoroughly studied yet, which should be further investigated in the future.

\section{Conclusions}

In this paper, the self-focusing condition of a charged particle beam in a resistive plasma has been studied. In the theoretical analysis, we started with the motion equation of the beam and found that the determinants of focusing are the beam thermal pressure $-\left(\mathrm{d} p_{\text {th }} / \mathrm{d} y\right)$ and the Lorentz force $J_{b} B_{x}$. The approximate focusing conditions in weak and strong Ohmic regimes are shown in inequalities (2.13) and (2.15). It is obtained that for weak Ohmic heating, increasing the beam density or velocity is good for focusing and for strong Ohmic heating, only increasing the beam velocity facilitates focusing. Furthermore, in the weak heating regime, the beam is focused to the centre as a whole, while in the strong heating regime, though the beam hollowing occurs near the centre, the beam can be focused away from the centre. It is also found that a larger beam radius will lead to a longer focusing distance. Then our theory was confirmed by the LAPINS code in the weak heating regime. Moreover, simulation results also show that if the beam radius is large enough, the ion beam filamentation phenomenon would occur, which should be further studied. Focusing and filamentation may occur in all kinds of phenomena associated with beam-plasma physics. Our results might serve as a reference for relevant beam-plasma experiments and theoretical analysis, such as heavy ion fusion, ion-beam-driven high energy density physics.

\section{Acknowledgements}

The authors are very grateful to the referees for their helpful suggestions and comments on improving the paper.

Editor Antoine C. Bret thanks the referees for their advice in evaluating this article. 


\section{Funding}

This work was supported by the National Natural Science Foundation of China (Z.M.S., grant numbers 11875235, 61627901), (D.W., grant number 12075204); National Magnetic Confinement Fusion Energy R\&D Program of China (Z.M.S., grant number 2019YFE03050001); Strategic Priority Research Program of Chinese Academy of Sciences (D.W., grant number XDA250050500); and Science Challenge Project (D.W., grant number TZ2016005).

\section{Declaration of interests}

The authors report no conflict of interest.

\section{Data availability statement}

The data that support the findings of this study are available from the corresponding authors upon reasonable request.

\section{REFERENCES}

Adli, E., Ahuja, A., Apsimon, O., Apsimon, R., Bachmann, A.-M., Barrientos, D., Batsch, F., Bauche, J., Berglyd Olsen, V.K., Bernardini, M., et al. 2018 Intrinsic stabilization of the drive beam in plasma-wakefield accelerators. Phys. Rev. Lett. 121, 064803.

ALfVÉn, H. 1939 On the motion of cosmic rays in interstellar space. Phys. Rev. 55, 425-429.

Allen, B., Yakimenko, V., Babzien, M., Fedurin, M., Kusche, K. \& Muggli, P. 2012 Experimental study of current filamentation instability. Phys. Rev. Lett. 109, 185007.

Bell, A.R., DAVIES, J.R. \& Guerin, S.M. 1998 Magnetic field in short-pulse high-intensity laser-solid experiments. Phys. Rev. E 58, 2471-2473.

Benford, G. 1973 Theory of filamentation in relativistic electron beams. Plasma Phys. 15, 483-499.

Bennett, W.H. 1934 Magnetically self-focussing streams. Phys. Rev. 45, 890-897.

Bennet, W.H. 1955 Self-focusing streams. Phys. Rev. 98, 1584-1593.

BERdANier, W., Roy, P.K. \& Kaganovich, I. 2015 Intense ion beam neutralization using underdense background plasma. Phys. Plasmas 22, 013104.

BRet, A., Firpo, M.C. \& Deutsch, C. 2005 Characterization of the initial filamentation of a relativistic electron beam passing through a plasma. Phys. Rev. Lett. 94, 115002.

Brown, L.S., Dean, L.P. \& Singleton, R.L. JR. 2005 Charged particle motion in a highly ionized plasma. Phys. Rep. 410, 237-333.

Caldwell, A., Lotov, K., Pukhov, A. \& Simon, F. 2018 Intrinsic stabilization of the drive beam in plasma-wakefield accelerators. Phys. Rev. Lett. 121, 064803.

Callahan, D.A. 1996 Chamber propagation physics for heavy ion fusion. Fusion Engng Des. 32-33, $441-452$.

Chen, B.Z., Wu, D., Ren, J.R., Hoffmann, D.H.H. \& ZhaO, Y.T. 2020 Transport of intense particle beams in large-scale plasmas. Phys. Rev. E 101, 051203.

Clauser, C.F. \& ARISTA, N.R. 2018 Stopping power of dense plasmas: the collisional method and limitations of the dielectric formalism. Phys. Rev. E 97, 023202.

Curcio, A. \& Volpe, L. 2019 A quasi-static model for hot-electron interaction with self-generated magnetic fields. Plasma Phys. Control. Fusion 61, 055013.

Davidson, R.C., Kaganovich, I., Qin, H., Startsev, E.A., Welch, D.R., Rose, D.V. \& Uhm, H.S. 2004 Collective instabilities and beam-plasma interactions in intense heavy ion beams. Phys. Rev. ST Accel. Beams 7, 114801.

DAVIES, J.R. 2003 Electric and magnetic field generation and target heating by laser-generated fast electrons. Phys. Rev. E 68, 056404.

Davies, J.R., Bell, A.R., Haines, M.G. \& GuéRin, S.M. 1997 Short-pulse high-intensity laser-generated fast electron transport into thick solid targets. Phys. Rev. E 56, 7193-7203.

Davies, J.R., Green, J.S. \& Norreys, P.A. 2006 Electron beam hollowing in laser-solid interactions. Plasma Phys. Control. Fusion 48, 1181-1199. 
Dorf, M.A., Davidson, R.C., Kaganovich, I.D. \& Startsev, E.A. 2012 Enhanced collective focusing of intense neutralized ion beam pulses in the presence of weak solenoidal magnetic fields. Phys. Plasmas 19, 056704.

Dorf, M.A., Kaganovich, I.D., Startsev, E.A. \& Davidson, R.C. 2009 Enhanced self-focusing of an ion beam pulse propagating through a background plasma along a solenoidal magnetic field. Phys. Rev. Lett. 103, 075003.

Dorf, M.A., Kaganovich, I.D., Startsev, E.A. \& Davidson, R.C. 2011 Collective focusing of intense ion beam pulses for high-energy density physics applications. Phys. Plasmas 18, 033106.

Frolova, A.A., Khishchenko, K.V. \& CHARAKHCH'YAn, A.A. 2019 Fast ignition by a proton beam and burning of a dt cylindrical shell target. Plasma Phys. Rep. 45, 830-849.

Govil, R., Leemans, W.P., Backhaus, E.Y. \& Wurtele, J.S. 1999 Observation of return current effects in a passive plasma lens. Phys. Rev. Lett. 83, 3202-3205.

HU, Z.H. \& WANG, Y.N. 2015 Self-pinched ion beam transport in plasmas with finite radius and nonuniform transverse density. Plasma Phys. Control. Fusion 57, 095003.

Kaganovich, I.D., Shvets, G., Startsev, E. \& Davidson, R.C. 2001 Nonlinear charge and current neutralization of an ion beam pulse in a pre-formed plasma. Phys. Plasmas 8, 4180-4192.

Kaganovich, I.D., Startsev, E.A., Sefkow, A.B. \& Davidson, R.C. 2008 Controlling charge and current neutralization of an ion beam pulse in a background plasma by application of a solenoidal magnetic field: Weak magnetic field limit. Phys. Plasmas 15, 103108.

Kim, J., McGuffey, C., Qiao, B., Wei, M.S., Grabowski, P.E. \& BeG, F.N. 2016 Varying stopping and self-focusing of intense proton beams as they heat solid density matter. Phys. Plasmas 23, 043104.

Lami, S.K., Kaphle, A.P., Briot, N.J., Botman, A. \& Hastings, J.T. 2020 Nanoscale focused electron beam induced etching of nickel using a liquid reactant. Nanotechnology 42, 425301.

LEE, R. \& LAMPE, M. 1973 Electromagnetic instabilities, filamentation, and focusing of relativistic electron beams. Phys. Rev. Lett. 31, 1390-1393.

Li, C.K. \& Petrasso, R.D. 1993 Charged-particle stopping powers in inertial confinement fusion plasmas. Phys. Rev. Lett. 70, 3059-3062.

Logan, B.G., Bieniosek, F.M., Celata, C.M., Coleman, J., Greenway, W., Henestroza, E., KWAn, J.W., LeE, E.P., Leitner, M., Roy, P.K., et al. 2007 Recent us advances in ion-beam-driven high energy density physics and heavy ion fusion. Nucl. Instrum. Meth. Phys. Res. A 577, 1-7.

Logan, B.G., PERKins, L.J. \& BARnARD, J.J. 2008 Direct drive heavy-ion-beam inertial fusion at high coupling efficiency. Phys. Plasmas 15 (7), 072701.

Martinez de la Ossa, A., Mehrling, T.J. \& OsterhofF, J. 2018 Intrinsic stabilization of the drive beam in plasma-wakefield accelerators. Phys. Rev. Lett. 121, 064803.

Norreys, P., Batani, D., Baton, S., Beg, F.N., Kodama, R., Nilson, P.M., Patel, P., Pérez, F., Santos, J.J., ScotT, R.H. H., et al. 2014 Fast electron energy transport in solid density and compressed plasma. Nucl. Fusion 54, 054004.

Ren, J., Deng, Z., Qi, W., Chen, B., MA, B., Wang, X., Yin, S., Feng, J., LiU, W., Xu, Z., et al. 2020 Observation of a high degree of stopping for laser-accelerated intense proton beams in dense ionized matter. Nat. Commun. 11, 5157.

Robertson, S. 1982 Collective focusing of an intense ion beam. Phys. Rev. Lett. 48, 149-151.

RoBinson, A.P.L., KEY, M.H. \& TABAK, M. 2012 Focusing of relativistic electrons in dense plasma using a resistivity-gradient-generated magnetic switchyard. Phys. Rev. Lett. 108, 125004.

Roth, M., Cowan, T.E., Key, M.H., Hatchett, S.P., Brown, C., Fountain, W., Johnson, J., Pennington, D.M., Snavely, R.A., Wilks, S.C., et al. 2001 Fast ignition by intense laser-accelerated proton beams. Phys. Rev. Lett. 86, 436-439.

Roy, P.K., Yu, S.S., Henestroza, E., Anders, A., Bieniosek, F.M., Coleman, J., Eylon, S., Greenway, W.G., Leitner, M., Logan, B.G., et al. 2005 Drift compression of an intense neutralized ion beam. Phys. Rev. Lett. 95, 234801.

Seidl, P.A., Anders, A., Bieniosek, F.M., Barnard, J.J., Calanog, J., Chen, A.X., Cohen, R.H., Coleman, J.E., Dorf, M., Gilson, E.P., et al. 2009 Progress in beam focusing and compression for warm-dense matter experiments. Nucl. Instrum. Meth. Phys. Res. A 606, 75-82. 
Sheng, L.N., Zhao, Y.T., Yang, G.J., Wei, T., Jiang, X.G., Zhou, X.M., Cheng, R., Yan, Y., LI, P., YANG, J.C., et al. 2014 Heavy-ion radiography facility at the institute of modern physics. Laser Part. Beams 32, 651-655.

SPITKOVSKY, A. 2007 On the structure of relativistic collisionless shocks in electron-ion plasmas. Astrophys. J. Lett. 673, L39-L42.

Tokluoglu, E.K., Kaganovich, I.D., Carlsson, J.A., Hara, K. \& Startsev, E.A. 2018 Amplification due to two-stream instability of self-electric and magnetic fields of an ion beam propagating in background plasma. Phys. Plasmas 25, 052122.

Turner, M., Adli, E., Ahuja, A., Apsimon, O., Apsimon, R., Bachmann, A.-M., Barros Marin, M., Barrientos, D., Batsch, F., Batkiewicz, J., et al. 2019 Experimental observation of plasma wakefield growth driven by the seeded self-modulation of a proton bunch. Phys. Rev. Lett. 122, 054801.

WANG, X.J., HU, Z.H. \& WANG, Y.N. 2020 Enhanced collective stopping and drift of electron beams in fusion plasmas with heavy-ion species. Phys. Rev. E 101, 043203.

Wu, D., He, X.T., Yu, W. \& Fritzsche, S. 2018 Particle-in-cell simulations of laser-plasma interactions at solid densities and relativistic intensities: the role of atomic processes. High Power Laser Sci. Engng 6, e50.

Wu, D., Yu, W., Fritzsche, S. \& He, X.T. 2020 Particle-in-cell simulation method for macroscopic degenerate plasmas. Phys. Rev. E 102, 033312.

Zylstra, A.B., Frenje, J.A., Grabowski, P.E., Li, C.K., Collins, G.W., Fitzsimmons, P., Glenzer, S., Graziani, F., Hansen, S.B., Hu, S.X., et al. 2015 Measurement of charged-particle stopping in warm dense plasma. Phys. Rev. Lett. 114, 215002. 